\section{DATES FOR THE DIARY}

Western Counties Branch AGM will take place on Monday 12 October at $6.30 \mathrm{pm}$. The venue is The Hankridge Arms, Riverside, Taunton, TA1 2LR (adjacent to J25 M5). To confirm your attendance, or submit apologies, please contact Alun Rees on alunrees@mac.com

The annual BASCD Joint Scientific and Consultant Group Meeting will take place on Thursday 19 November at Cavendish House, London. The theme for the conference will be 'action on free sugars - what is BASCD's view? The day will feature high-profile speakers in the morning and an afternoon drafting a position statement.

\section{0\% GET DFT PLACES FOR $2^{\text {nd }}$ YEAR RUNNING}

The British Dental Association (BDA) has welcomed confirmation from COPDEND that every eligible UK graduate has secured a dental foundation training (DFT) place for the second year running.

The announcement covers DFT places in England, Wales and Northern Ireland. The BDA has campaigned hard on the issue, highlighting how the historic lack of places had hurt dental graduates, and represented a poor use of public money invested in training unsuccessful applicants.

Judith Husband, Chair of the BDA's Education, Ethics and the Dental Team Working Group, said: 'For the second year running every UK dental graduate has secured a training place. This makes sense, for the next generation of dentists and for the public purse. We shouldn't expect taxpayers to pick up the tab giving graduates skills they might never get the chance to use as young dentists.

'We were determined that last year would not be remembered as a "oneoff”. This year's result is great news. Now we have to make sure it becomes a tradition.'

\title{
CHILDREN IN SPECIAL SCHOOLS HAVE MORE SEVERE TOOTH DECAY COMPARED TO CHILDREN IN MAINSTREAM SCHOOLS
}

Children in special support schools have slightly lower levels of tooth decay than children in mainstream schools but are more likely to have their teeth extracted, according to a new survey published today (24 September 2015) by Public Health England (PHE).

The first national survey of oral health in special schools - schools for children with severe special education needs and disabilities (SEND) - also show that those who experienced decay have more teeth affected and their oral hygiene is poorer. The number of children in special support schools with a substantial amount of plaque is double that of those attending mainstream schools (4\% and 2\% respectively for 5-year-olds and 20\% and 10\% respectively for 12-year-olds).

The survey, broken down by region, shows wide variation in tooth decay prevalence and severity ranging from $10 \%$ to $33 \%$ for 5 -year-olds and $22 \%$ to $41 \%$ for 12 -year-olds. The North West region has the poorest dental health for both age groups in special support schools.

Dr Sandra White, Director of Dental Public Health at PHE, said: 'Despite children in special support schools having slightly lower levels of tooth decay than children in mainstream schools, they are still very high so we must not be complacent. Children in special support schools are particularly vulnerable so they require an additional package of support to prevent and treat tooth decay.'

\section{STOPTOBER RETURNS TO GIVE QUITTERS A HEALTHY SMILE}

Stoptober - Public Health England's (PHE) 28 day mass quit smoking campaign - has launched as the most recent statistics reveal 2,790 new cases of oral cancer were diagnosed in England in 2013. On average an estimated 65\% (70\% in males and 55\% in females) of oral cancer cases are linked to smoking tobacco.

PHE is urging dental professionals to engage in Stoptober by offering 'Very Brief Advice' (VBA) to smokers using a '30 second approach'; a technique developed by The National Centre for Smoking Cessation and Training, and further referenced by PHE in their resources for dental professionals. When compared with no advice to smokers, recommending treatment or support via VBA has been shown to increase the odds of quitting by $68 \%$ and $217 \%$ respectively.

VBA includes three simple steps:

- ASK, establish and record smoking status

- ADVISE on the personal benefits of quitting in light of oral health findings

- ACT, offer help and signpost to Stoptober and local Stop Smoking Services.

Research shows that by stopping smoking for 28 days, people are five times more likely to stop for good. Now in its fourth year, the campaign is targeted at all smokers with a particular emphasis on routine and manual workers, who have high rates of smoking. Stoptober offers a variety of free tools including encouraging and supportive emails, text messages or the Stoptober mobile phone app - all of which have shown to increase the chances of staying smokefree.

Supporting the campaign, Dr Sandra White, Director of Dental Public Health for Public Health England, said: 'Smokers are seven to ten times more likely to suffer from oral cancer than individuals who have never smoked. Smoking can increase the chances of gum disease and tooth loss; it leads to stained teeth, reduced taste sensation and halitosis.

'Whilst dental professionals have a brief opportunity to engage with smokers about their plans for quitting, the "Very Brief Advice" approach is designed to be used in almost any consultation with a smoker or tobacco user. VBA is a simple and straightforward way in which dental teams can support their patients to quit tobacco. We'd urge all dental practitioners to get involved in this year's campaign to encourage even more people to quit for 28 days and beyond.'

Sara Hurley, Chief Dental Officer, NHS England, said: 'It is a sad reality that one in two of us will get a form of cancer at some point in our lives with the likelihood of contracting mouth cancer heightened by excess drinking and smoking. Stoptober is another great opportunity for dentists to reinforce prevention messages and encourage patients to take up Stoptober. The dental team can motivate their patients by highlighting all the oral health benefits of stopping smoking - reducing risk of tooth loss, halitosis and gum disease - signposting to local stop smoking services and by providing that extra layer of support and encouragement to patients trying to give up.'

Dental professionals can find further information regarding the 'Very Brief Advice' technique in Delivering Better Oral Health: an evidence-based toolkit and Smokefree and Smiling, helping dental patients to quit tobacco. 